\title{
A High Order Characteristic Discontinuous Galerkin Scheme for Advection on Unstructured Meshes
}

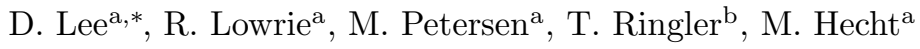 \\ ${ }^{a}$ Computer, Computational and Statistical Sciences, Los Alamos National Laboratory, Los Alamos, \\ NM 87545, USA \\ ${ }^{b}$ Theoretical Division, Los Alamos National Laboratory, Los Alamos, NM 87545, USA
}

\section{Abstract}

A new characteristic discontinuous Galerkin (CDG) advection scheme is presented. In contrast to standard discontinuous Galerkin schemes, the test functions themselves follow characteristics in order to ensure conservation and the edges of each element are also traced backwards along characteristics in order to create a swept region, which is integrated in order to determine the mass flux across the edge. Both the accuracy and performance of the scheme are greatly improved by the use of large Courant-FriedrichsLewy numbers for a shear flow test case and the scheme is shown to scale sublinearly with the number of tracers being advected, outperforming a standard flux corrected transport scheme for 10 or more tracers with a linear basis. Moreover the CDG scheme may be run to arbitrarily high order spatial accuracy and on unstructured grids, and is shown to give the correct order of error convergence for piecewise linear and quadratic bases on regular quadrilateral and hexahedral planar grids. Using a modal Taylor series basis, the scheme may be made monotone while preserving conservation with the use of a standard slope limiter, although this reduces the formal accuracy of the scheme to first order. The second order scheme is roughly as accurate as the incremental remap scheme with nonlocal gradient reconstruction at half the horizontal resolution. The scheme is being developed for implementation within the Model for Prediction Across Scales (MPAS) Ocean model, an unstructured grid finite volume ocean model.

Keywords: Discontinuous Galerkin, Advection equation, High order advection, Lagrangian characteristics, Unstructured grid

\section{Introduction}

Tracer advection constitutes a large portion of the compute time for modern global climate models, due to the large number of chemical and hydrometeor species that must be accounted for. For physical consistency, the advection of tracers must be conservative while being as numerically accurate and computationally efficient as possible. The

${ }^{*}$ Corresponding author. Tel. +1 5056657286

Email address: drlee@lanl.gov (D. Lee) 
preservation of monotonicity may also be required. Here we present a novel characteristic discontinuous Galerkin (CDG) advection scheme which allows for arbitrarily long time steps and monotonic, conservative advection while also scaling sub-linearly with the advection of additional tracers such that it outperforms a traditional flux corrected transport (FCT) scheme [1] for a moderate number of tracers. The scheme may be implemented at arbitrarily high order, through the use of a modal basis expansion of the tracer in each element.

Several recent developments in conservative, monotonic schemes have been presented and implemented for the advection of tracers with large Courant-Friedrichs-Lewy (CFL) numbers and sublinear computational costs for large numbers of tracers. Lagrangian incremental remapping (IR) schemes have been presented on both planar Cartesian [2] and spherical geodesic [3] grid. In such schemes the vertices of each edge of an element are traced backwards along velocity characteristics, and a piecewise linear reconstruction of the tracer at the previous time level is used to integrate the intersection between the swept region made by the edge and its departure points and the neighbouring elements at the previous time level. The mean tracer values in the neighbouring elements are then used to reconstruct the linear representation of the tracer in the element at the new time level, and these gradients may be limited to preserve monotonicity. The swept regions are common to all tracers and so only need be computed once for each edge at each time level. The resulting second order scheme is shown to out-perform a standard FCT scheme for more than seven tracers on a spherical geodesic geometry [3].

A similar scheme has been implemented for a cubed sphere geometry, the Conservative Semi-Lagrangian Multi-tracer transport scheme (CSLAM) [4, 5] by which the elements, rather than the edges, are advected back along characteristics. The advection problem is then solved in remap form with the intersecting regions between the pre-image of the element and the Eulerian grid at the previous time level integrated using line integrals via the Gauss-Green theorem in order to determine the weights of a quadratic polynomial representation of the tracer in each element. This scheme may in theory be extended to arbitrarily high order and is currently in use within the High-Order Methods Modelling Environment (HOMME) atmospheric model [6]. Another scheme implemented within the HOMME model, Spectral Element Lagrangian Transport (SPELT) [7] uses a quadratic polynomial reconstruction of the tracer within each element to integrate the flux across the Lagrangian pre-image of the element at intermediate time levels to third order spatial accuracy. Like the CSLAM algorithm, this scheme uses line integrals instead of swept regions for greater computational efficiency, however the nature of the tracer field reconstructions limits the scheme to quadrilateral grids.

The methods discussed above use some form of reconstruction to determine the higher order structure of the tracer field. An alternative approach is to introduce a set of test functions which are integrated along velocity characteristics so to satisfy the adjoint equation to the weak form of the problem. This is the approach used in the EulerianLagrangian Localized Adjoint Method (ELLAM) [8, 9], which has been used to solve transport, advection-diffusion and reactive problems for groundwater flows.

One downside of the ELLAM method is that it requires the assembly and solution of a global system of equations, as either a finite volume or finite element problem. This issue is negated in a similar and recently developed semi-Lagrangian discontinuous Galerkin scheme for tracer transport in atmospheric flows on cubed spheres [10], which prognoses the trial function representation of the tracer, while integrating the quadrature points 
of the test functions forwards in time along velocity characteristics in order to satisfy the adjoint equation. The method is applied in one dimension, for which the quadrature points of the Lagrangian pre-image of the element are integrated forward in time, where they are used to evaluate the tracer via its trial function representation. This is required in order to preserve the values of the test functions along characteristics, as required by the method. The method is applied to the cubed sphere geometry by way of a second order in time Strang operator splitting, by which one dimension is evolved a half step in time, then the second dimension a full step, then the first dimension another half step. The method is conservative due to the exact nature of the quadrature integration and accurate to arbitrarily high spatial order, but limited to Cartesian topologies due to the splitting scheme.

The characteristic discontinuous Galerkin (CDG) scheme discussed in this paper is similar to the previous semi-Lagrangian discontinuous Galerkin scheme [10] in that the higher order structure is prognosed via the solution of a system of linear equations for the coefficients of the trial functions in each element, with the fluxes determined via an integration of the swept region made by the vertices of the edges along characteristics. However unlike the previous scheme it is applied in two dimensions without the use of dimensional splitting, and so is suitable for fully unstructured grids. Moreover because it is two dimensional, the temporal order of accuracy is not limited by the operator splitting. The CDG scheme is being developed for future use in the Model for Prediction Across Scales (MPAS) Ocean model [11], a mimetic finite volume model, since it is anticipated to be more computationally efficient than the existing FCT scheme for large numbers of tracers.

The remainder of this paper is presented as follows: In Section 2 the formulation of the CDG scheme for the advection equation is presented, including a comparison to standard discontinuous Galerkin (DG) methods and discussions of the scheme's conservation and stability properties. Section 3 describes the implementation of the algorithm in the discrete form. Section 4 presents the results of various numerical experiments and comparisons to another characteristic based advection scheme, incremental remap (IR). These comparisons include convergence rates with varying orders of trial and test functions, and with varying CFL number, for a divergence-free shear flow and the computational cost of the scheme with respect to an increasing number of tracers and comparisons to a standard FCT scheme, as well as convergence rates for a monotone version of the scheme via slope limiting. In section 5 the conclusions are discussed.

\section{Formulation}

\subsection{Standard discontinuous Galerkin advection}

The advection of a tracer $q$ by velocity $\vec{u}$ is given in flux form as

$$
\frac{\partial q}{\partial t}+\nabla \cdot(\vec{u} q)=0 .
$$

According to the standard discontinuous Galerkin formulation $[12,13,14],(1)$ is solved for a set of $k$ contiguous elements $\Omega_{k}$ which span the full domain $\Omega$. These are multiplied by a set of piecewise smooth test functions $\beta_{k, i}(\vec{x})$, and integrated over each element $k$, to give the weighted residual expression 


$$
\frac{\mathrm{d}}{\mathrm{d} t}\left(\int_{\Omega_{k}} \beta_{k, i} q \mathrm{~d} \Omega_{k}\right)+\int_{\Omega_{k}} \beta_{k, i} \nabla \cdot(\vec{u} q) \mathrm{d} \Omega_{k}=0
$$

by which the residual error of the discrete approximation to (1) is known to be zero over the domain $\Omega$. Integrating by parts and applying Gauss' theorem gives the weak form of the problem as

$$
\frac{\mathrm{d}}{\mathrm{d} t}\left(\int_{\Omega_{k}} \beta_{k, i} q \mathrm{~d} \Omega_{k}\right)=\int_{\Omega_{k}} \nabla \beta_{k, i} \cdot \vec{u} q \mathrm{~d} \Omega_{k}-\int_{\partial \Omega_{k}} \beta_{k, i} \vec{u} q \cdot \mathrm{d} \vec{s}
$$

where $\partial \Omega_{k}$ is the boundary of element $k$ and $\vec{s}$ is the normal vector to the element boundary. If we assume that the tracer is similarly approximated by a set of $j$ trial functions in each element and that these trial functions are of the same form as the test functions such that

$$
q_{k}(\vec{x}, t) \approx \sum_{j} c_{k, j}(t) \beta_{k, j}(\vec{x})
$$

then we arrive at the Galerkin formulation of the problem

$$
\frac{\mathrm{d}}{\mathrm{d} t}\left(\sum_{j} \int_{\Omega_{k}} \beta_{k, i} \beta_{k, j} \mathrm{~d} \Omega_{k} c_{k, j}(t)\right)=\int_{\Omega_{k}} \nabla \beta_{k, i} \cdot \vec{u} q \mathrm{~d} \Omega_{k}-\int_{\partial \Omega_{k}} \beta_{k, i} \vec{u} q \cdot \mathrm{d} \vec{s} .
$$

For a detailed discussion of the method of weighted residuals and Galerkin formulations for advection equations the reader is referred to the standard texts [15, 14].

If continuity is not enforced for the trial functions across element boundaries, then (5) may be solved independently for each element, such that a global mass matrix becomes unnecessary. The boundary fluxes may then be approximated as some average of the values evaluated from the trial functions of the elements either side of the edge. This is known as the discontinuous Galerkin method. Equation (5) is commonly solved explicitly using a Runge-Kutta time integration scheme $[12,13,14]$, which is subject to a CFL time stepping constraint proportional to $1 /(2 l+1)$, where $l$ is the order of the basis functions and $l+1$ is the order of the Runge-Kutta scheme.

\subsection{Characteristic discontinuous Galerkin advection}

An alternative to the standard discontinuous Galerkin formulation, which is not subject to a CFL number constraint, is to instead multiply $q$ in (1) by a set of test functions which vary in both space and time $\phi_{k, i}(\vec{x}, t)$, and expand via the product rule as

$$
\frac{\partial \phi_{k, i} q}{\partial t}+\nabla \cdot\left(\phi_{k, i} \vec{u} q\right)=\phi_{k, i}\left(\frac{\partial q}{\partial t}+\nabla \cdot(\vec{u} q)\right)+q\left(\frac{\partial \phi_{k, i}}{\partial t}+\vec{u} \cdot \nabla \phi_{k, i}\right) .
$$

The first term on the right hand side of (6) is identical to the original advection equation (1) and so vanishes, and the second term represents the material derivative of the test functions $D \phi_{k, i} / D t$. Integrating by parts over the element area $\Omega_{k}$ and between time levels $n$ and $n+1$ and applying Gauss' theorem, the weak form is given as 


$$
\begin{aligned}
\int_{\Omega_{k}}\left(\phi_{k, i} q\right)^{n+1}-\left(\phi_{k, i} q\right)^{n} \mathrm{~d} \Omega_{k}+\int_{t^{n}}^{t^{n+1}} \int_{\partial \Omega_{k}} \phi_{k, i} \vec{u} q \cdot \mathrm{d} \vec{s} \mathrm{~d} t & = \\
& \int_{t^{n}}^{t^{n+1}} \int_{\Omega_{k}} q \frac{D \phi_{k, i}}{D t} \mathrm{~d} \Omega_{k} \mathrm{~d} t .
\end{aligned}
$$

The right hand side may be taken as zero if the values of the test functions are constant along characteristics, a condition also enforced in the ELLAM $[8,9]$ and semi-Lagrangian discontinuous Galerkin [10] schemes, such that

$$
\frac{D \phi_{k, i}}{D t}=0 .
$$

Equation (8) is satisfied via the introduction of a test function $\beta$ which varies with respect to a Lagrangian coordinate in space and time $\vec{\Gamma}$ as

$$
\phi_{k, i}(\vec{x}, t)=\beta_{k, i}(\vec{\Gamma}(\vec{\xi}(s), s))
$$

where $\vec{\Gamma}(\vec{\xi}(s), s)$ is constant with respect to the parametric variable $s$ along the characteristic trajectory

$$
\frac{\mathrm{d} \vec{\xi}}{\mathrm{d} s}=\vec{u}(\vec{\xi}(s), s) \quad \vec{\xi}(t)=\vec{x}
$$

and $t$ is the point on $s$ where the boundary condition is applied, such that

$$
\frac{\mathrm{d} \vec{\Gamma}(\vec{\xi}(s), s)}{\mathrm{d} s}=0 \quad \vec{\Gamma}\left(\vec{x}, t^{n+1}\right)=\vec{\xi}\left(t^{n+1}\right) .
$$

Note that the boundary condition for (11) follows from that for (10) such that for any $s=t, \Gamma(\vec{\xi}(t), t)=\Gamma(\vec{x}, t)$, with $t^{n+1}$ being the specific time at which the boundary condition is applied.

In order to show that (8) is indeed satisfied we begin by applying the chain rule as

$$
\frac{D \phi(\vec{x}, t)}{D t}=\frac{\mathrm{d} \beta(\vec{\Gamma}(\vec{\xi}(t), t))}{\mathrm{d} t}=\nabla_{\vec{\Gamma}} \beta(\vec{\Gamma}(\vec{\xi}(t), t)) \frac{\mathrm{d} \vec{\Gamma}(\vec{\xi}(t), t)}{\mathrm{d} t}
$$

where $\nabla_{\vec{\Gamma}}=\left(\partial / \partial \Gamma_{x}, \partial / \partial \Gamma_{y}\right)$ with $t$ held constant, such that we need only determine the path of $\vec{\Gamma}$ in Eulerian coordinates $(\vec{x}, t)$ for which $\mathrm{d} \vec{\Gamma}(\vec{x}, t) / \mathrm{d} t=0$. Integrating (10) with respect to $s$ between $t$ and $t^{n+1}$ and recalling the boundary conditions on $\vec{\xi}(t)$ and $\vec{\xi}\left(t^{n+1}\right)$ gives

$$
\vec{\Gamma}(\vec{x}, t)=\vec{x}+\int_{t}^{t^{n+1}} \vec{u}(\vec{\xi}(s), s) \mathrm{d} s
$$

such that for any $(\vec{x}, t)(13)$ preserves the constant value of $\beta$ along characteristics and hence the constant value of $\phi$ along those same characteristics.

Equation (13) implies that the test functions arrive at their static Eulerian coordinates $\vec{x}$ at time level $n+1$ such that $\phi_{k, i}\left(\vec{x}, t^{n+1}\right)=\beta_{k, i}(\vec{x})$, with $\vec{x}$ being the location of 
$\vec{\Gamma}\left(\vec{x}, t^{n+1}\right)$ as given in (13). The values of the test functions at the same coordinate at the previous time level $n$ are then given as $\phi_{k, i}\left(\vec{x}, t^{n}\right)=\beta_{k, i}\left(\vec{\Gamma}\left(\vec{x}, t^{n}\right)\right)$. Taking the simple example of a characteristic moving with respect to a constant velocity $\vec{u}$ over a single time step $\Delta t=t^{n+1}-t^{n}$, such that $\Gamma\left(\vec{x}, t^{n}\right)=\vec{x}+\vec{u} \Delta t$, we have $\phi\left(x, t^{n}\right)=\beta(\vec{x}+\vec{u} \Delta t)$, such that the test functions move with the velocity field along the characteristics. Again recalling (13) we see from the above example that $\vec{\Gamma}\left(\vec{x}, t^{n+1}\right)=\vec{\Gamma}\left(\vec{x}, t^{n}\right)-\vec{u} \Delta t$, such that unlike the test functions, the characteristics actually move backwards in time with respect to the Eulerian coordinates and velocity. As a practical matter, this means that if we wish to evaluate a test function $\phi_{k, i}\left(\vec{x}, t^{n}\right)$ at a given coordinate $\vec{x}$ at a previous time level $n$ subject to (8) then we may equivalently evaluate the static test function $\beta_{k, i}\left(\vec{\Gamma}\left(\vec{x}, t^{n}\right)\right)$ at its previous location by integrating forwards with the Eulerian velocity field.

As for the standard discontinuous Galerkin formulation, the tracer field may be approximated by a series of trial functions in each element as given in (4). Unlike the test functions, $\phi_{k, i}(\vec{x}, t)$, there is no requirement that these trial functions be conserved along characteristics, and so may remain static, defined at the arrival locations of the characteristics at time level $n+1$ as given in (13). Ensuring that the mass matrix by which the solution coefficients are multiplied remained static in this fashion motivated our choice of boundary conditions in (10) and (11). This gives rise to the following linear system for the solution of the trial function coefficients $c_{k, j}^{n+1}$ in each element $k$ at the new time level $n+1$

$$
\sum_{j} \int_{\Omega_{k}} \beta_{k, i} \beta_{k, j} \mathrm{~d} \Omega_{k} c_{k, j}^{n+1}=\int_{\Omega_{k}}\left(\phi_{k, i} q\right)^{n} \mathrm{~d} \Omega_{k}-\int_{t^{n}}^{t^{n+1}} \int_{\partial \Omega_{k}} \phi_{k, i} \vec{u} q \cdot \mathrm{d} \vec{s} \mathrm{~d} t .
$$

The solution of (14) subject to (8) represents the characteristic discontinuous Galerkin formulation for updating the tracer trial function coefficients $c_{k, j}^{n+1}$ at the new time level $n+1$. As discussed the mass matrix operator on the left hand side of (14) is constant for all time and the same for all tracers. For each element it therefore needs only be assembled and its inverse calculated once and stored for use in determining the trial function coefficients at each successive time step.

Like the standard discontinuous Galerkin formulation given in (5), only the boundary fluxes are required to determine the solution of the tracer coefficients at the new time level, so no global mass matrix is required. However unlike the standard discontinuous Galerkin formulation, where the fluxes are determined via some Eulerian process, such as some form of averaging, upwinding or a Riemann solver, under the CDG formulation the edge fluxes must be evaluated by taking the area made by the edge as it is swept backward in time to its static Eulerian location from the previous time level and integrating the tracer mass over this area. This may be expressed as

$$
\sum_{j} \int_{\Omega_{k}} \beta_{k, i} \beta_{k, j} \mathrm{~d} \Omega_{k} c_{k, j}^{n+1}=\int_{\Omega_{k}}\left(\phi_{k, i} q\right)^{n} \mathrm{~d} \Omega_{k}-\sum_{f} \int_{\Delta \Omega_{k, f}}\left(\phi_{k, i} q\right)^{n} s_{k, f} \mathrm{~d} \Delta \Omega_{k, f}
$$

where $\Delta \Omega_{k, f}$ is the swept region made by edge $f$ of element $k$, and $s_{k, f}$ is the sign of the flux across this face with respect to element $k$. A discussion of how this swept region may be evaluated, and how (8) may be enforced is given in the subsequent section 
on implementation. The flux-form representation of the CDG scheme (15) also ensure conservation of $q$, which will be discussed in more detail in Section 2.4.

\subsection{Remap $C D G$}

In addition to the flux form implementation of the CDG scheme given in (15), (14) may also be evaluated in remap form, by which the intersections of the Eulerian mesh and the pre-images of the elements at the previous time level, rather than the swept regions of the edges, are evaluated to determine the coefficients of the trial functions at the new time level. The remap version of the CDG scheme for updating the coefficients of the trial functions for element $k$ at time level $n+1$ may be expressed as

$$
\sum_{j} \int_{\Omega_{k}} \beta_{k, i} \beta_{k, j} \mathrm{~d} \Omega_{k} c_{k, j}^{n+1}=\sum_{l} \int_{\Omega_{k, l}} \phi_{k, i}^{n} q^{n} \mathrm{~d} \Omega_{k, l}
$$

where $\Omega_{k, l}$ denotes the set of $l$ intersection regions between the pre-image of element $k$ integrated back along velocity characteristics to time level $n$ and its neighbour elements $l$ on the static Eulerian mesh. Note that as for the flux form given in (14), the remap form of the CDG scheme also requires that test functions $\phi$ be constant along characteristics in order to satisfy (8).

\subsection{Conservation}

In order to demonstrate the local conservation of (14) over element $k$ we begin by assuming a set of coefficients $a_{i}$ for which

$$
\sum_{i} a_{i} \beta_{k, i}(\vec{x})=1
$$

Multiplying (15) by these same coefficients and summing over $i$ gives

$$
\begin{aligned}
& \sum_{i} a_{i} \sum_{j} \int_{\Omega_{k}} \beta_{k, i} \beta_{k, j} \mathrm{~d} \Omega_{k} c_{k, j}^{n+1}=\sum_{i} a_{i} \int_{\Omega_{k}}\left(\phi_{k, i} q\right)^{n} \mathrm{~d} \Omega_{k}- \\
& \sum_{i} a_{i} \sum_{f} \int_{\Delta \Omega_{k, f}}\left(\phi_{k, i} q\right)^{n} s_{k, f} \mathrm{~d} \Delta \Omega_{k, f} .
\end{aligned}
$$

Recalling (17) and (9) this reduces to

$$
\int_{\Omega_{k}}\left(q^{n+1}-q^{n}\right) \mathrm{d} \Omega_{k}-\sum_{f} \int_{\Delta \Omega_{k, f}} q^{n} s_{k, f} \mathrm{~d} \Delta \Omega_{k, f} .
$$

Assuming that the same quadrature points are used to approximate the integral over the element $\Omega_{k}$ at all time levels, then local conservation in time is assured when these integrals are replaced by quadrature since

$$
\int_{\Omega_{k}}\left(q^{n+1}-q^{n}\right) \mathrm{d} \Omega_{k}+\int_{\Omega_{k}}\left(q^{n}-q^{n-1}\right) \mathrm{d} \Omega_{k}=\int_{\Omega_{k}}\left(q^{n+1}-q^{n-1}\right) \mathrm{d} \Omega_{k}
$$

For the integration of the flux across face $f$ between elements $k$ and $k^{\prime}$ we have 


$$
\sum_{f} \int_{\Delta \Omega_{k, f}} q^{n} s_{k, f} \mathrm{~d} \Delta \Omega_{k, f}=-\sum_{f} \int_{\Delta \Omega_{k^{\prime}, f}} q^{n} s_{k^{\prime}, f} \mathrm{~d} \Delta \Omega_{k^{\prime}, f}
$$

which is balanced since the flux is heading in opposing directions with respect to $k$ and $k^{\prime}$ such that $s_{k, f}=-s_{k^{\prime}, f}$. The CDG scheme as given in (15) is therefore shown to be locally conservative in both space and time for a set of basis functions which are a partition of unity, as in (17), which true for common choices, including the basis discussed in Section 2.6.

Note that the above argument for conservation is made only for the flux based CDG scheme given in (15) and not the remap based scheme given in (16). As is the case for other remapping advection schemes [5], this form of the CDG scheme should also be conservative, though this is not proven here.

\subsection{Stability}

In this section we provide a proof for the stability of the remap formulation of the CDG scheme for a constant velocity field. While the transformation between the remap and flux forms of the CDG scheme should hold for the discrete approximation of these forms if the quadrature is exact, the following should not be regarded as a rigorous proof of the stability of the flux form of the CDG scheme with variable velocity field.

We begin by multiplying (16) by the solution coefficients $c_{k, i}^{n+1}$ and summing over $i$ and $j$ on the left hand side such that

$$
\int_{\Omega_{k}}\left(q\left(\vec{x}, t^{n+1}\right)\right)^{2} \mathrm{~d} \Omega_{k}=\sum_{l} \int_{\Omega_{k, l}} \sum_{i} c_{k, i}^{n+1} \phi_{k, i}\left(\vec{x}, t^{n}\right) q\left(\vec{x}, t^{n}\right) \mathrm{d} \Omega_{k, l} .
$$

${ }_{25}$ Recalling (9) the right hand side may be reformulated such that

$$
\int_{\Omega_{k}}\left(q\left(\vec{x}, t^{n+1}\right)\right)^{2} \mathrm{~d} \Omega_{k}=\sum_{l} \int_{\Omega_{k, l}} q\left(\vec{\Gamma}\left(\vec{x}, t^{n}\right), t^{n+1}\right) q\left(\vec{x}, t^{n}\right) \mathrm{d} \Omega_{k, l} .
$$

Summing globally over all elements $k$ and assuming that the pre-image of all the elements at the previous time level contiguously span the entire domain we have

$$
\int_{\Omega}\left(q\left(\vec{x}, t^{n+1}\right)\right)^{2} \mathrm{~d} \Omega=\int_{\Omega} q\left(\vec{\Gamma}\left(\vec{x}, t^{n}\right), t^{n+1}\right) q\left(\vec{x}, t^{n}\right) \mathrm{d} \Omega
$$

where $\Omega$ is the global domain made up of the set of $k$ contiguous elements. Invoking the Cauchy-Schwarz inequality gives

$$
\left(\int_{\Omega}\left(q\left(\vec{x}, t^{n+1}\right)\right)^{2} \mathrm{~d} \Omega\right)^{2} \leq\left(\int_{\Omega}\left(q\left(\vec{\Gamma}\left(\vec{x}, t^{n}\right), t^{n+1}\right)\right)^{2} \mathrm{~d} \Omega\right)\left(\int_{\Omega}\left(q\left(\vec{x}, t^{n}\right)\right)^{2} \mathrm{~d} \Omega\right) .
$$

Given that the velocity field is constant, the Jacobian transformation between $\vec{x}$ and $\vec{\Gamma}$ is unity, such that if we are integrating over the full domain then

$$
\int_{\Omega}\left(q\left(\vec{\Gamma}\left(\vec{x}, t^{n}\right), t^{n+1}\right)\right)^{2} \mathrm{~d} \Omega=\int_{\Omega}\left(q\left(\vec{x}, t^{n+1}\right)\right)^{2} \mathrm{~d} \Omega
$$


such that

$$
\int_{\Omega}\left(q\left(\vec{x}, t^{n+1}\right)\right)^{2} \mathrm{~d} \Omega \leq \int_{\Omega}\left(q\left(\vec{x}, t^{n}\right)\right)^{2} \mathrm{~d} \Omega .
$$

Equation (27) states that the remap formulation of the CDG scheme is stable for any time step under the strong assumption that the velocity field is constant in time. Note that this does not guarantee stability for the flux based formulation of the scheme or for variable velocity fields. It should also be noted that the scheme may become unstable if the characteristics traced back from the vertices of a given edge intersect one another yielding a bowtie swept region. This will occur if the condition $|\nabla \vec{u}|_{\max } \Delta t<1$ is violated $[2]$.

\subsection{Basis functions}

Nothing has been said yet about the form of the basis functions, other than that they are piecewise continuous within each element. For the implementation discussed below we use a set of $i$ modal Taylor series basis functions for each element $k$ defined as

$$
\beta_{k, i}(x, y)=\frac{1}{(n-m) ! m ! \Delta x^{n-m} \Delta y^{m}}\left(x^{n-m} y^{m}-\bar{x}_{k}^{n-m} \bar{y}_{k}^{m}\right) \quad i=\sum_{p=0}^{n} p+m \quad m \leq n
$$

$$
\bar{x}_{k}^{n-m} \bar{y}_{k}^{m}=\frac{1}{A_{k}} \int_{\Omega_{k}} x^{n-m} y^{m} \mathrm{~d} \Omega_{k} .
$$

where $A_{k}$ is the area of cell $k$. Each of the higher order terms of the basis function is scaled to ensure that these terms remain $\mathcal{O}(1)$. This ensures that the condition number of the mass matrix assembled from these terms and described in the proceeding section remains moderate. Note that $(x, y)$ is defined with respect to the origin of the local coordinate system of element $k$.

\section{Implementation}

In order to satisfy (8) when solving (14) at each time step, the quadrature points used to evaluate the test functions during the assembly of the right hand side are integrated forward in time to their arrival points at the new time level, as is also the case for the semi-Lagrangian discontinuous Galerkin scheme [10]. The temporal integration of the flux term on the right hand side of (14) also requires the integration of the vertices of each edge backwards in time along velocity characteristics in order to determine the swept region of the mass flux across an edge within a given time step. This flux may then be evaluated by breaking the swept region up into triangles and integrating the mass of the tracer in each triangle using the Fekete quadrature points [16, 15] of an order consistent with that of the trial and test functions for exact integration. The use of triangular 
quadrature for both the swept regions and the target elements allows for the application of the CDG scheme to arbitrary unstructured grids of $n$-sided convex polygons, which may be broken up into $n$ triangles. All quadrature and vertex characteristic integration is performed using second order Runge-Kutta. An algorithm for assembling the right hand side flux vector is presented below.

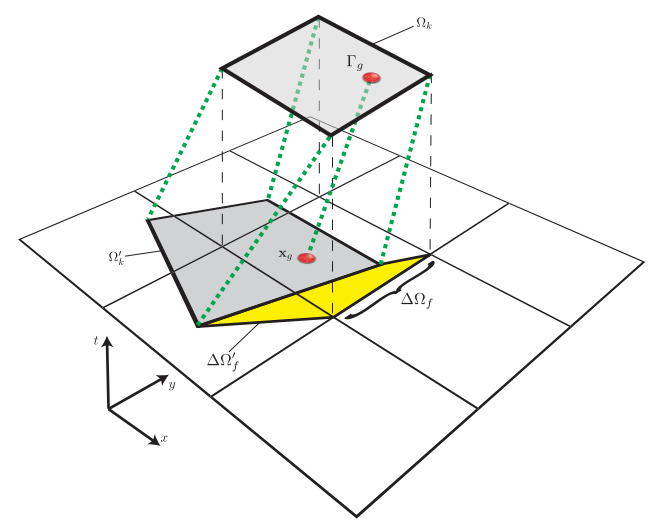

Figure 1: Schematic of the flux computation for the CDG scheme. Edge vertices are integrated back in time to their departure points in order to determine the swept region for an edge over a given time step. The volume of the tracer over the swept region is then integrated with the quadrature points integrated forwards to arrival points where the test functions are evaluated in order to preserve the value of the test functions along characteristics.

- Integrate the vertices backwards in time along velocity characteristics.

- For each edge:

1. Determine the element for which the flux is heading into, $k+$.

2. Determine the element for which the flux is heading from, $k-$.

3. For each element in the region of the edge determined by the CFL number:

(a) Determine the intersection polygon between the edge pre-image and the neighbouring element.

(b) Break the intersection polygon into a set of triangles, $t r$.

(c) Generate quadrature points for each of the triangles.

(d) For each quadrature point $\vec{x}_{t r, p}$ :

i. Loop over all tracers $l$ and evaluate the $q_{l}\left(\vec{x}_{t r, p}, t^{n}\right)$.

ii. Integrate $\vec{x}_{t r, p}$ forwards in time along velocity characteristics to its arrival point $\vec{x}_{t r, p}^{*}$.

iii. Evaluate each test function at $\vec{x}_{t r, p}^{*}$ for element $k+, \phi_{k+, i}\left(\vec{x}_{t r, p}^{*}\right)$.

iv. Evaluate each test function at $\vec{x}_{t r, p}^{*}$ for element $k-, \phi_{k-, i}\left(\vec{x}_{t r, p}^{*}\right)$.

v. Loop over all tracers $l$ and add the area weighted product of $q_{l}\left(\vec{x}_{t r, p}, t^{n}\right)$ and $\phi_{k+, i}\left(\vec{x}_{t r, p}^{*}\right)$ to the flux vector of element $k+$.

vi. Loop over all tracers $l$ and subtract the area weighted product of $q_{l}\left(\vec{x}_{t r, p}, t^{n}\right)$ and $\phi_{k-, i}\left(\vec{x}_{t r, p}^{*}\right)$ to the flux vector of element $k-$.

Note that the advection of additional tracers only requires one additional loop to evaluate, while the evaluation of the swept regions and bases is the same, thus reducing the computational cost of advecting additional tracers. 


\subsection{Element assembly}

The operators in (14) may be assembled using the Fekete quadrature points as follows

$$
\begin{aligned}
\int_{\Omega_{k}} \beta_{k, i} \beta_{k, j} \mathrm{~d} \Omega_{k} & \approx \sum_{t c} A_{t c} \sum_{p} w_{p} \phi_{k, i}\left(\vec{x}_{t c, p}\right) \phi_{k, j}\left(\vec{x}_{t c, p}\right) \\
\int_{\Omega_{k}}\left(\phi_{k, i} q\right)^{n} \mathrm{~d} \Omega_{k} & \approx \sum_{t c} A_{t c} \sum_{p} w_{p} \phi_{k, i}\left(\vec{x}_{t c, p}^{*}\right) q\left(\vec{x}_{t c, p}, t^{n}\right) \\
\int_{t^{n}}^{t^{n+1}} \int_{\partial \Omega_{k}} \phi_{k, i} \vec{u} q \cdot \mathrm{d} \vec{s} \mathrm{~d} t & \approx \sum_{e} \sum_{k^{\prime}} \int_{\Omega_{k^{\prime}, e}} \phi_{k, i} q \mathrm{~d} \Omega_{k^{\prime}, e} \\
& \approx \sum_{e} \sum_{k^{\prime}} \sum_{t i} A_{e, k^{\prime}, t i} n_{e, k^{\prime}, t i} \sum_{p} w_{p} \phi_{k, i}\left(\vec{x}_{e, k^{\prime}, t i, p}^{*}\right) q\left(\vec{x}_{e, k^{\prime}, t i, p}, t^{n}\right)
\end{aligned}
$$

where $k^{\prime}$ is the set of elements neighbouring $k, \Omega_{k^{\prime}, e}$ is the intersection of the swept region made by edge $e$ over element $k^{\prime}, A_{t c}$ is the area of triangle $t c$ for element $k, A_{e, k^{\prime}, t i}$ is the area of the triangle $t i$ for $\Omega_{k^{\prime}, e}, n_{e, k^{\prime}, t i}$ is the direction of the flow with respect to the normal vector of edge $e$ for triangle $t i( \pm 1), w_{p}$ is the dimensionless weight of quadrature point $p, \vec{x}_{t c, p}$ is the position of quadrature point $p$ in triangle $t c, \vec{x}_{t c, p}^{*}$ is its arrival point, $\vec{x}_{e, k^{\prime}, t i, p}$ is the position of quadrature point $p$ in triangle $t i$ of the intersection region of the pre-image of edge $e$ and neighbouring element $k^{\prime}$ and $\vec{x}_{e, k^{\prime}, t i, p}^{*}$ is its arrival point.

Note that $q(\vec{x}, t)$ is always evaluated by the expansion of the trial functions with respect to the coefficients $c_{k^{\prime}, i}$ of the element in which $\vec{x}$ is found, irrespective of whether $k^{\prime}$ is the target element $k$ for which the solution is being determined or not. Note also that the direction of the flow with respect to the edge may differ for different triangles of the intersection region of the edge pre-image. This is because the departure points of the edge may be located on different sides of the edge, leading to a bowtie intersection polygon, for which part of the mass flux heads in one direction though the edge and part in the opposite direction.

\subsection{Quadrature}

The coordinates of the quadrature points $\vec{x}_{t r, q}$ for a given triangle $t r$ are generated as a product of the Fekete quadrature point barycenters $f_{v, q}[16,15]$ and the geometric vertices $v$ of the triangle $\vec{x}_{t r, v}$ as

$$
x_{t r, q}=\sum_{v=1}^{3} x_{t r, v} f_{v, q}-x_{k} \quad y_{t r, q}=\sum_{v=1}^{3} y_{t r, v} f_{v, q}-y_{k}
$$

where $\vec{x}_{k}=\left(x_{k}, y_{k}\right)$ is the origin of element $k$.

\subsection{Monotonicity}

Monotonicity may be enforced for the CDG scheme on an unstructured grid with a linear basis by the use of a van Leer [17, 18], vertex based Barth-Jespersen [19, 20] or multi-dimensional limiting process [21]. Such approaches can ensure against overshoots at element boundaries, however for the application of a higher order basis they cannot 
guarantee that the internal representation of the tracer field within each element will remain monotonic. To maintain higher order accuracy for the limited solution with internal extrema, some form of hierarchical Barth-Jespersen limiter may be applied [20]. However here we only seek to demonstrate the amenability of the CDG scheme to monotone limiting and make no attempt to present a limiting strategy that preserves the higher order accuracy of the monotone solution. Due to the requirement that the CDG scheme work on arbitrary unstructured grids, standard WENO limiters [22] which rely on some knowledge of the element topology cannot be applied.

Here the vertex-based Barth-Jespersen limiter is applied [20], where the linear terms in $(28)$ are scaled by a factor $\alpha=\min \left(\alpha_{v}\right)$ such that

$$
\tilde{q}(\vec{x}, t)=c_{k, 0}^{n}+\alpha\left(c_{k, 1}^{n} \beta_{k, 1}(\vec{x})+c_{k, 2}^{n} \beta_{k, 2}(\vec{x})\right)
$$

where $\tilde{q}$ is the monotone form of the tracer and $\alpha_{v}$ is the limiting factor determined at the vertices $v$ of element $k$, defined as

$$
\alpha_{v}= \begin{cases}\left(c_{k^{\prime}, 0}^{\max }-c_{k, 0}\right) /\left(q_{k}\left(\vec{x}_{v}\right)-c_{k, 0}\right) & \text { if } q_{k}\left(\vec{x}_{v}\right)>c_{k, 0} \\ 1 & \text { if } q_{k}\left(\vec{x}_{v}\right)=c_{k, 0} \\ \left(c_{k^{\prime}, 0}^{\min }-c_{k, 0}\right) /\left(q_{k}\left(\vec{x}_{v}\right)-c_{k, 0}\right) & \text { if } q_{k}\left(\vec{x}_{v}\right)<c_{k, 0} .\end{cases}
$$

Here $k^{\prime}$ denotes the set of elements that share vertex $v$ with element $k$ (including itself), and $c_{k^{\prime}, 0}^{\max / \min }$ are the maximum and minimum mean values of the tracer on $k^{\prime}, \vec{x}_{v}$ is the coordinate of the vertex $v$, and $q_{k}\left(\vec{x}_{v}\right)$ is the value of the tracer evaluated at vertex $v$ using the solution coefficients of element $k, c_{k, i}$. Recall from (28) that the integrals of the higher order terms over a given element are 0 , such that $c_{k, 0}$ represents the mean value of $q$ over the element, such that limiting these higher order terms does not result in a loss of conservation. Note also that if the limiter is applied for a given element (such that $\alpha<1$ ), then all the coefficients for the quadratic and higher terms in the trial function are set to 0 . This is because strict monotonicity within the element cannot be guaranteed for higher order terms with the application of the limiter. Elements for which the limiter is applied are thus formally only first order accurate.

\section{Results}

\subsection{Spatial convergence}

The CDG scheme has been tested for a time varying deformation-rotation velocity field [23] of the form

$$
u_{\theta}=\frac{4 \pi r}{T}\left(1-\cos \left(\frac{2 \pi t}{T}\right)\right)\left(\frac{1-(4 r)^{6}}{1+(4 r)^{6}}\right) \quad u=u_{\theta} \sin (\theta) \quad v=-u_{\theta} \cos (\theta)
$$

where $r=\sqrt{(x-1)^{2}+(y-1)^{2}}$ and $\theta=\tan ^{-1}((y-1) /(x-1))$ for $0 \leq x, y \leq 2$ and a single period of $T=30000$. This velocity field represents a robust test of the advection scheme as it involves a shearing of the initial tracer field, which returns to its original position at both $t=T / 2$ and $t=T$ via different trajectories, without a reversal of the velocity field which would mask phase errors for the Fourier modes of the tracer field. Convergence plots have been generated on both regular quadrilateral and hexahedral 
grids with resolutions of $\Delta x=0.0625,0.03125,0.015625$ and 0.0078125 (where $\Delta x$ represents the distance between two neighbouring element centers), and a constant CFL number of $\left|u_{\theta}\right| \Delta t / \Delta x \approx 0.1$. A Gaussian hill initial condition of the form

$$
q(x, y, t=0)=e^{\left(-320\left(\left(x-x_{0}\right)^{2}+\left(y-y_{0}\right)^{2}\right)\right)} \quad x_{0}=1+\sqrt{2} / 4 \quad y_{0}=1+\sqrt{2} / 4
$$

is chosen since it is infinitely differentiable and so allows us to demonstrate the convergence of the CDG scheme at higher order.

The initial condition $q(x, y, t=0)$ is projected onto the trial functions for the tracer in each element $k$ in order to determine their initial coefficients $c_{k, j}^{0}$ by solving the linear system

$$
\sum_{j} \int_{\Omega_{k}} \beta_{k, i} \beta_{k, j} \mathrm{~d} \Omega_{k} c_{k, j}^{0}=\int_{\Omega_{k}} \beta_{k, i} q^{0} \mathrm{~d} \Omega_{k}
$$

The $L_{2}$ errors are presented in Figure 3 for linear and quadratic bases on both quadrilateral and hexahedral grids. These are calculated as

$$
L_{2}=\sum_{\Omega_{k}} \frac{\int_{k}\left(q-q_{A}\right)^{2} \mathrm{~d} \Omega_{k}}{\int_{\Omega_{k}} q_{A}^{2} \mathrm{~d} \Omega_{k}}
$$

where $q_{A}=q(x, y, t=0)$ is the analytic solution after a period $T$ as given in (34) and $q$ is evaluated as in (4). The convergence rates are determined by taking the logarithm of the $L_{2}$ errors and the corresponding horizontal resolutions $\Delta x$ and fitting a least squares linear trend to this. As can be seen, the scheme converges as the formal order of accuracy suggests for both the linear and quadratic bases on both grid topologies.

While third order accuracy has been achieved for the test presented here, it should be noted that for general cases this requires that the pre-image of the edge be integrated backwards along the velocity characteristics using three spatial locations and that the departure points of these locations be fitted to a quadratic curve and that this be integrated exactly. This issue has been addressed for the CSLAM scheme by parameterizing the curved edges and integrating in the parametric coordinates [24]. In future work this issue will be addressed, most likely using similar methods.

\subsection{Variable CFL number}

The scheme has also been shown to be stable and convergent for large CFL numbers (greater than 1). Figure 4 gives the convergence rates for the linear basis CDG scheme on the hexahedral grid with a velocity field of $\vec{u}=(+\mathrm{U} \sin (k x) \cos (k y),-\mathrm{U} \cos (k x) \sin (k y))$, $\mathrm{U}=2.5 \pi \times 10^{-5}, k=\pi / 2$ which is reversed at $T / 2=20000$ and the same initial condition and resolutions as described above for CFL numbers of 0.1, 0.4, 1.25 and 2.5. For larger CFL numbers intersections must be determined between the swept region and a deeper set of elements in the vicinity of the edge, not just its immediate neighbours as is the case with a CFL of less than 1 . The $L_{2}$ errors are found to decrease for larger CFL numbers, signifying that the growth in temporal truncation error due to larger time step size is more than compensated for by the reduction in error due to the lower frequency with which the swept region fluxes and quadrature point integrations must be approximated. Note that this trend may be expected to continue with the use of time 


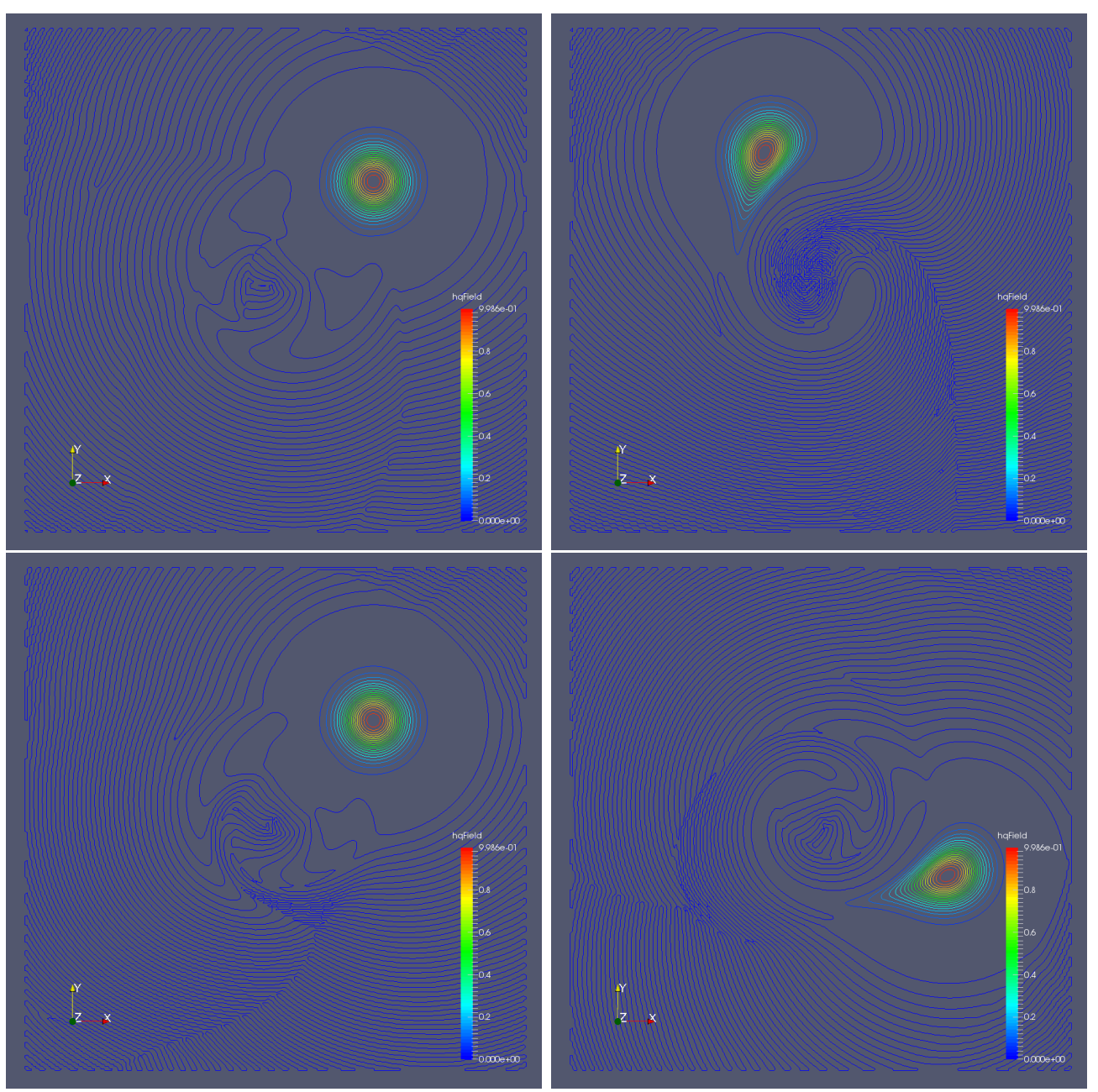

Figure 2: Tracer field $q$ at $t=T$ (top left), $t=T / 4$ (top right), $t=T / 2$ (bottom left) and $t=3 T / 4$ (bottom right) for the quadrilateral grid with $\Delta x=0.0078125$ 

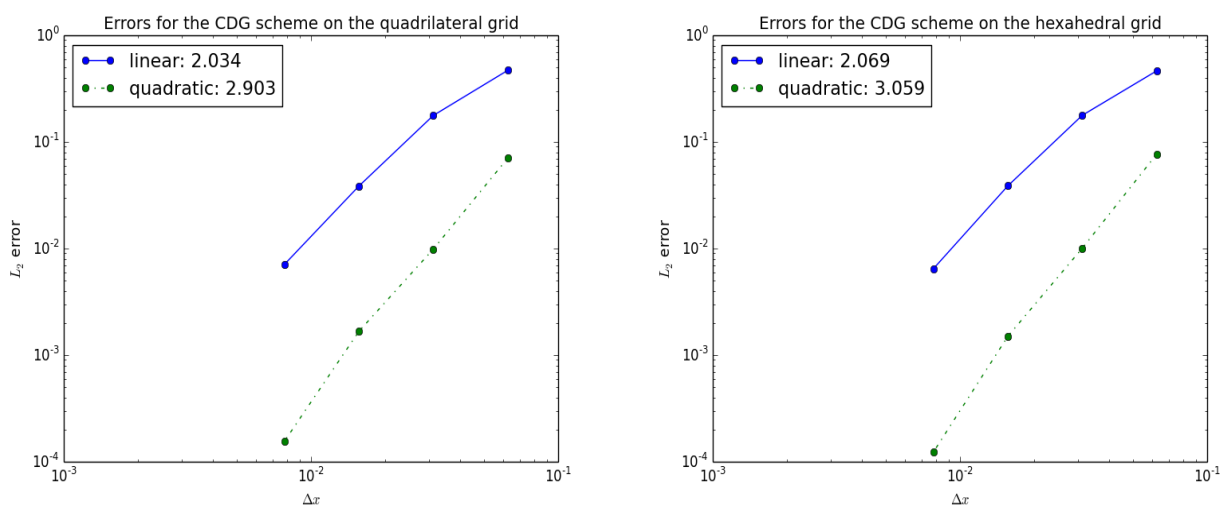

Figure 3: Convergence rates for the linear and quadratic bases on quadrilateral (left) and hexahedral (right) grids.

integrators of higher order than the second order Runge-Kutta scheme used here. This increase in accuracy with CFL number has also been reported previously for the CSLAM characteristic advection scheme [4]. In addition to being more accurate, the CDG scheme is approximately $20 \%$ faster for a doubling of the CFL number. This suggests that the expense associated with a larger halo region and more intersection and quadrature point integrations per time step for a larger CFL number is more than offset by the reduction in the total number of time steps.
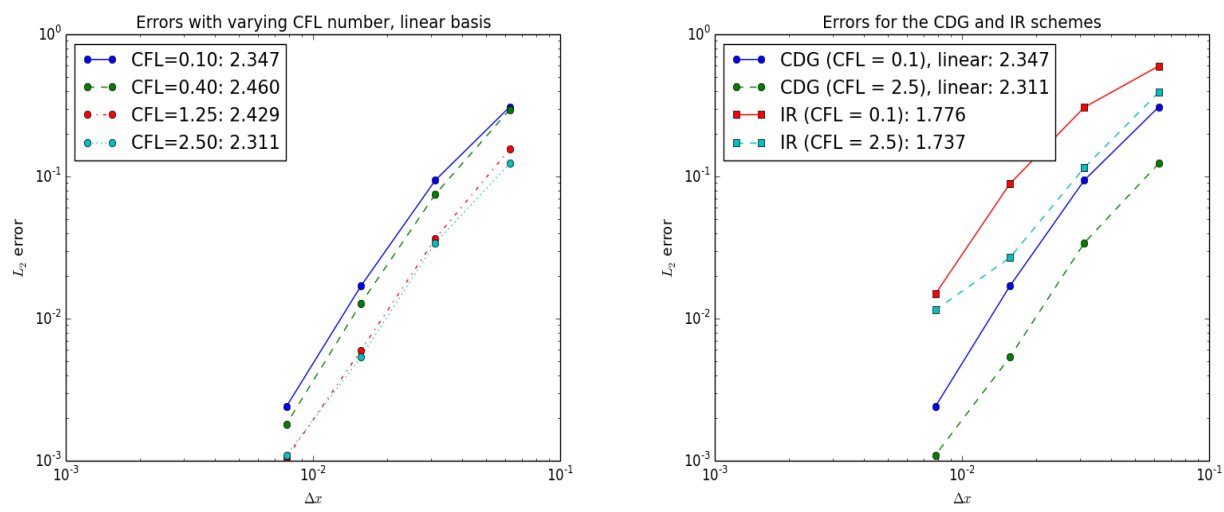

Figure 4: Convergence rates for the linear basis CDG scheme at varying CFL numbers of 0.1, 0.4, 1.25 and 2.5 (left) and comparison to IR with CFL numbers of 0.1 and 2.5 (right).

Figure 4 also shows a comparison between linear CDG and the characteristic-based second order incremental remap (IR) advection scheme [2] for CFL numbers of 0.1 and 2.5. As for the CDG scheme, fluxes for the IR scheme are determined via the integration of swept regions of the edges along characteristics, and so the IR scheme also appears to be stable for large CFL numbers. The principle difference between the CDG and IR 
schemes is in the method used to determine the higher order moments. In the CDG scheme these are represented by trial functions which, along with the mean components in each element, are evolved prognostically with the fluxes. In the IR scheme however, the gradients are reconstructed from the mean values of the tracer in the target element and its neighbours. Notably, for a given CFL number the CDG scheme is approximately as accurate as the IR scheme with twice the horizontal resolution. This is most likely because the CDG trial functions are local to the given element and therefore compact, while the IR gradients are determined from the mean values of the tracer in the neighbouring elements.

\subsection{Scaling of compute time with number of tracers}

The execution times have also been evaluated for the linear CDG and incremental remapping schemes [2] with varying numbers of tracers and CFL number, in comparison with those for a standard FCT scheme. These timings are performed for a grid of resolution $\Delta x=0.03125$ using 16 AMD Opteron model 6176 processors with an individual clock speed of $2.3 \mathrm{GHz}$ on a single node. The linear basis CDG, IR and FCT schemes were run with a CFL of 0.1 , and then the CDG and IR schemes were rerun with a CFL of 2.5. This larger CFL number allows for a reduced number of time steps, while requiring a larger area of element intersections be calculated for each swept region. Gradients were calculated by taking a least squares fit of the logarithm of both the compute time and number of tracers. The resulting gradients give a measure of the additional computational expense associated with a doubling of the number of tracers being advected.
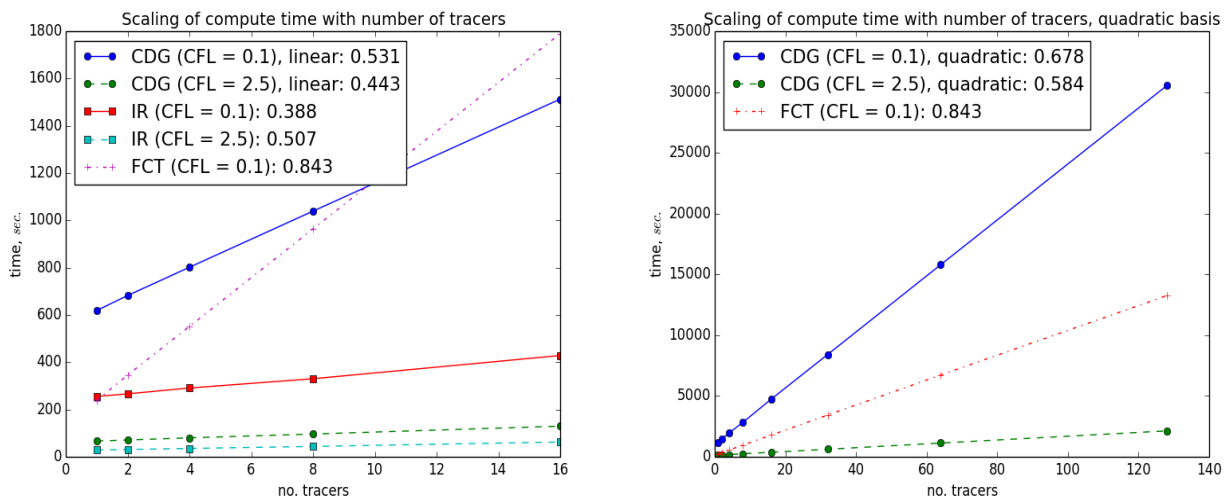

Figure 5: Scaling of compute time with number of tracers for the linear CDG and IR schemes at CFL numbers of 0.1 and 2.5, and the FCT scheme with CFL $=0.1$ (left) and quadratic CDG with CFL numbers of 0.1 and 2.5 and FCT again with $\mathrm{CFL}=0.1$ (right).

As can be seen in Figure 5, the FCT scheme scales at a rate of approximately 1 for a doubling in the number of tracers being advected. This is to be expected, since the advection of each additional tracer is independent of every other, while the linear basis CDG scheme with $\mathrm{CFL}=0.1$ has an additional expense of $53 \%$ with a doubling in the number of tracers and with a significant start up cost due to the computation of the 
swept regions and the integration of quadrature points. The CDG scheme with a CFL of 0.1 is more computationally efficient than the FCT scheme for approximately 10 tracers. The IR scheme meanwhile is significantly more efficient, with a reduced start up cost compared to the CDG scheme, and a lower computational cost than the FCT scheme for two or more tracers.

The comparative cost of the CDG scheme may be reduced greatly by the use of a larger CFL number. Note however that this saving may be reduced somewhat by the increased halo size required for each edge, and the associated parallel communication required. On a single node, the CDG scheme with a CFL of 2.5 is more efficient than the FCT scheme for a single tracer, with an additional cost of $44 \%$ for a doubling in the number of tracers. The same is also true for the IR scheme, which is still more efficient than the CDG scheme.

Figure 5 also shows the compute times for the quadratic CDG scheme with increased tracer counts. With a CFL of 0.1 the quadratic scheme scales almost as poorly as the FCT scheme, with an additional expense of $68 \%$ for a doubling in the number of tracers, however this cost is reduced greatly with an increased CFL number, such that with a CFL of 2.5 the quadratic scheme is more efficient than the FCT scheme while being an order more accurate.

The additional expense of the CDG scheme compared to the IR scheme may be attributed to the relative cost of assembling and solving a system of linear equations for each element and the integration of quadrature points for the CDG scheme, compared to the reconstruction of gradients from neighbouring element mean values as done in the IR scheme. Note that as this computation is local and requires no additional parallel communication, the relative cost of the CDG scheme may potentially be reduced by porting some of these operations to accelerators present on advanced computing architectures in situations where there is latency in parallel communication across the machine.

\subsection{Monotonicity}

The monotone scheme has been tested for the time varying deformation-rotation velocity field (33) on the hexahedral grid. This involves the application of the slope limiter given in (31) and (32), with the coefficients of the quadratic basis terms set to 0 wherever $\alpha<1$ in order to suppress any internal extrema within a given element. As can be seen from Figure 6, the monotone scheme reduces the accuracy significantly, with the monotone quadratic CDG scheme converging at a similar rate to the non-monotone linear scheme.

\section{Conclusion}

The formulation of a conservative high order advection scheme for unstructured grids has been presented. This characteristic discontinuous Galerkin (CDG) scheme has been shown to converge at its formal order of accuracy for linear and quadratic modal bases on both regular quadrilateral and hexahedral grids. The scheme has been shown theoretically to be unconditionally stable with time-step for constant flows in remap form, and numerical experiments of the flux form implementation demonstrate that not only is the scheme stable with large CFL numbers for divergence-free shear flow, but that the accuracy of the scheme increases with time step. With a CFL of 0.1 the linear basis CDG 


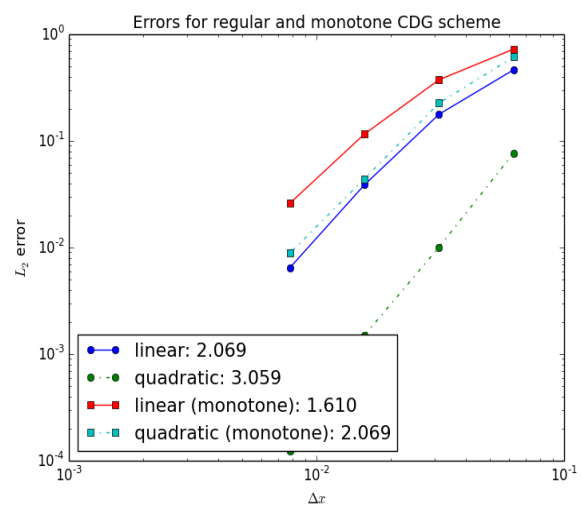

Figure 6: Convergence errors for the slope limited monotone CDG scheme on linear and quadratic bases.

scheme is $53 \%$ more expensive for a doubling in the number of tracers being advected, and more efficient than the FCT scheme for the advection of 10 tracers and beyond. With a CFL of 2.5, the CDG scheme is more efficient than the FCT scheme for a single tracer, and $44 \%$ more expensive for a doubling in the number of tracers. Using a CFL of 2.5 also improves the performance of the quadratic CDG scheme to the point where it is more efficient than the FCT scheme as well as being an order more accurate.

While the incremental remap scheme was found to be more computationally efficient than the CDG scheme, and has a superior cost saving with an increased number of tracers, the linear basis CDG scheme is approximately as accurate as the IR scheme with double the horizontal resolution. We believe this to be because the stencil used to determine the gradients in the IR scheme encompasses the neighbouring elements, while the higher order trial functions are all local to the element for the CDG scheme, such that the same information is effectively determined with a smaller stencil (a single element).

The scheme may be made monotone with the application of a slope limiter appropriate for unstructured grids, however the application of the limiter greatly reduces the accuracy of the scheme. Also the slope limiter applied in this study [20] cannot account for internal maxima within each element which may occur for quadratic and higher order bases, and so these are zeroed out wherever the limiter is applied.

In future work the scheme will be implemented on the sphere for use in the MPASOcean model [11]. This will require a method for projecting from the planar space of the target element to the sphere that is consistent with the formal order of accuracy of the basis, so as to not corrupt the rate of error convergence on the sphere. Additional work will be undertaken to investigate the use of vertical advection schemes that are consistent with the horizontal CDG advection scheme without introducing additional truncation errors, as are inherent in the dimensional splitting of the prior semi-Lagrangian discontinuous Galerkin scheme [10].

\section{Acknowledgements}

The authors are grateful to Dr. William Lipscomb for his assistance regarding the implementation of the incremental remap algorithm and to Dr. Doug Jacobsen for his 
advice regarding the implementation of the CDG scheme within the MPAS framework. We would also like to acknowledge the support of LANL Institutional Computing. This work was carried out under the auspices of the National Nuclear Security Administration of the U.S. Department of Energy at Los Alamos National Laboratory under Contract No. DE-AC52-06NA25396. This research was supported by the Office of Science (BER), U.S. Department of Energy. Los Alamos Report LA-UR-16-22694. Matthew Hecht, as coauthor, was supported through the HiLAT project of the Regional and Global Climate Modeling program of the DOE's Office of Science.

\section{References}

[1] J. P. Boris, D. L. Book, Flux-corrected transport. i. shasta, a fluid transport algorithm that works, J. Comp. Phys. 11 (1973) 38-69.

[2] J. K. Dukowicz, J. R. Baumgardner, Incremental remapping as a transport/advection algorithm, J. Comp. Phys. 160 (2000) 318-335.

[3] W. H. Lipscomb, T. D. Ringler, An incremental remapping transport scheme on a spherical geodesic grid, Mon. Wea. Rev. 133 (2005) 2335-2350.

[4] P. H. Lauritzen, R. D. Nair, P. A. Ullrich, An conservative semi-lagrangian multi-tracer transport scheme (CSLAM) on the cubed-sphere grid, J. Comp. Phys. 229 (2010) 1401-1424.

[5] C. Erath., P. H. Lauritzen, H. M. Tufo, On mass conservation in high-order high-resolution rigorous remapping schemes on the sphere, Mon. Wea. Rev. 141 (2013) 2128-2133.

[6] M. A. Taylor, A. Fournier, An compatible and conservative spectral element method on unstructured grids, J. Comp. Phys. 229 (2010) 5879-5895.

[7] C. Erath, R. D. Nair, A conservative multi-tracer transport scheme for spectral-element spherical grids, J. Comp. Phys. 256 (2014) 118-134.

[8] M. A. Celia, T. F. Russell, I. Herrera, R. E. Ewing, An eulerian-lagrangian localized adjoint method for the advection-diffusion equation, Adv. Water Resources 13 (1990) 187-206.

[9] T. F. Russell, M. A. Celia, An overview of research on eulerian-lagrangian localized adjoint methods (ELLAM), Adv. Water Resources 25 (2002) 1215-1231.

[10] W. Guo, R. D. Nair, J.-M. Qiu, A conservative semi-lagrangian discontinuous galerkin scheme on the cubed sphere, Mon. Wea. Rev. 142 (2014) 457-475.

[11] T. Ringler, M. Petersen, R. L. Higdon, D. Jacobsen, P. W. Jones, M. Maltrud, A multi-resolution approach to global ocean modeling, Ocean Modelling 69 (2013) 211-232.

[12] B. Cockburn, S. Hou, C.-W. Shu, The runge-kutta local projection discontinuous galerkin finite element method for conservation laws iv: The multidimensional case, Math. Comp. 54 (1990) 545581.

[13] B. Cockburn, C.-W. Shu, Runge-kutta discontinuous galerkin methods for convection-dominated problems, J. Sci. Comput. 16 (2001) 173-261.

[14] J. S. Hesthaven, T. Warburton, Nodal Discontinuous Galerkin Methods, Algorithms, Analysis, and Applications, Springer, 2008

[15] G. E. Karniadakis, S. Sherwin, Spectral/hp Element Methods for Computational Fluid Dynamics, Oxford University Press, 2005.

[16] M. A. Taylor, B. A. Wingate, R. E. Vincent, An algorithm for computing fekete points in the triangle, SIAM J. Numer. Anal. 38 (2000) 1707-1720.

[17] B. van Leer, Towards the ultimate conservative difference scheme. v. a second-order sequel to godunov's method, J. Comp. Phys. 32 (1979) 101-136.

[18] J. K. Dukowicz, J. W. Kodis, Accurate conservative remapping (rezoning) for arbitrary lagrangianeulerian computations, SIAM J. Sci. Stat. Comput. 8 (1987) 305-321.

[19] T. Barth, D. C. Jespersen, The design and application of upwind schemes on unstructured meshes, Tech. Rep. AIAA Paper 89-0366 (1989).

[20] D. Kuzmin, A vertex-based hierarchical slope limiter for $p$-adaptive discontinuous galerkin methods, J. Comp. Appl. Math. 233 (2010) 3077-3085.

[21] J. S. Park, C. Kim, Higher-order multi-dimensional limiting strategy for discontinuous galerkin methods in compressible inviscid and viscous flows, Computers \& Fluids 96 (2014) 377-396.

[22] C. Hu, C.-W. Shu, Weighted essentially non-oscillatory schemes on triangular meshes, J. Comp. Phys. 150 (1999) 97-127. 
571 [23] P. N. Blossey, D. R. Durran, Selective monotonicity preservation in scalar advection, J. Comp. $572 \quad$ Phys. 227 (2008) 5160-5183.

573 [24] P. A. Ullrich, P. H. Lauritzen, C. Jablonowski, Some considerations for high-order 'incremental remap'-based transport schemes: edges, reconstructions, and area integration, Int. J. Numer. Meth. Fluids 71 (2013) 1131-1151. 